\title{
TREATMENT OF CUTANEOUS TUMORS WITH TOPICAL 5\% IMIQUIMOD CREAM
}

\author{
Sabrina Sisto Alessi, Jose Antonio Sanches, Walmar Roncalli de Oliveira, Maria \\ Cristina Messina, Eugenio Raul de Almeida Pimentel, Cyro Festa Neto
}

doi: $10.1590 / \mathrm{S1807-59322009001000005}$

\begin{abstract}
Alessi SS, Sanches JÁ, Oliveira WR, Messina MC, Pimentel ERA, Neto CF. Treatment of cutaneous tumors with topical 5\% imiquimod cream. Clinics. 2009;64(10):961-6.
\end{abstract}

INTRODUCTION: There are various approaches to the treatment of cutaneous tumors; one of them is treatment with imiquimod, a synthetic toll-like receptor agonist with a low molecular weight that offers a topical, noninvasive, and non-surgical therapeutic option. The main objective of our study was to provide data on 89 patients who used a 5\% imiquimod cream for the treatment of cutaneous tumors at the Cutaneous Oncology Group of the Dermatology Department of Hospital das Clinicas from 2003 to 2008. MATERIALS AND METHODS: Here, we present our experience in the treatment of 123 cutaneous tumors of various types, including basal cell carcinoma (BCC), squamous cell carcinoma (SCC), Bowen's disease, erythroplasia of Queyrat, Paget's disease, and trichoepithelioma, with 5\% imiquimod cream from 2003 to 2008 in the Cutaneous Oncology Group of the Dermatology Department of Hospital das Clinicas. Patients were divided into two separate groups according to their diagnosis and comorbidities; these comorbidities included epidermodysplasia verruciformis, xeroderma pigmentosum, albinism, basal cell nevus syndrome, Brooke-Spiegler syndrome, HIV, chronic lymphocytic leukemia, B-cell lymphoma, and kidney transplantation. Treatment duration, response to imiquimod, follow-up, recurrence, and local and systemic reactions associated with use of the drug were analyzed. Epidemiological data were obtained and cure rates were calculated.

RESULTS: The ratio of women to men was 1.28:1, and the mean age was 63.1 years. Tumors were located mainly on the face, back, trunk, and legs. For patients with comorbidities, the overall cure rate was $38 \%$. These specific patients demonstrated cure rates of $83.5 \%$ for superficial BCC and 50\% for Bowen's disease. Aggressive BCC and superficial and nodular BCC did not present a good response to treatment. Trichoepitheliomas and nodular BCC showed a partial response, and erythroplasia of Queyrat showed a complete response. For patients without comorbidities, the overall cure rate was $73 \%$. For these patients, the cure rates were $85.7 \%$ for superficial and nodular BCC, $88 \%$ for superficial BCC, 57\% for Bowen's disease, 50\% for nodular BCC, and 50\% for aggressive BCC. One SCC lesion demonstrated a complete response, and tumors caused by Paget's disease and erythroplasia of Queyrat presented a partial response. None of the tumors considered as clinically cured recurred. Thirty-seven lesions demonstrated no response to imiquimod. Having a cutaneous comorbidity, high-risk tumors such as mixed aggressive BCC (sclerodermiform or micronodular), nodular BCC, or Bowen's disease, and presenting no local reaction to imiquimod were considered as risk factors for a worse prognosis. We demonstrate that patients with no response to imiquimod, even when they demonstrated no local reaction, can undergo another cycle of six weeks of imiquimod treatment and show a complete response. The healing pattern led to good cosmetic outcomes, and the side effects were tolerable.

CONCLUSIONS: Our experience confirms imiquimod as an effective treatment option for several types of cutaneous tumors, especially in patients without the cutaneous comorbidities cited above and with low-risk tumors. Imiquimod has a relatively low cost compared to other therapeutic options and can be delivered via ambulatory care to patients with surgery contraindications, and its side effects are tolerable.

KEYWORDS: Non-melanoma skin cancer; Imiquimod; Immunomodulator; Basal cell carcinoma; Immunotherapy.

Dermatology Department, Hospital das Clinicas da Faculdade de Medicina da Universidade de São Paulo - São Paulo/SP, Brazil.

Tel.: 55113081.2551

Email: saurin_89@hotmail.com

Received for publication on May 21, 2009

Accepted for publication on July 13, 2009

\section{INTRODUCTION}

Imiquimod is capable of enhancing both innate and cellmediated immune pathways. It stimulates the innate immune response through the induction, synthesis, and release of 
cytokines. Imiquimod activates antigen-presenting cells such as monocytes, macrophages, B cells, and dendritic cells by activating toll-like receptors (TLRs), which are a family of pathogen recognition receptors. The stimulation of TLRs results in the activation of a signaling cascade that recruits protein kinases and transcription factors, ultimately promoting maturation and the secretion of interleukins (ILs) 12 and 18 and interferon-alpha (IFN- ) by target cells. The secretion of these cytokines induces the secretion of interferon-gamma (IFN-) by naive $\mathrm{T}$ cells, resulting in the development of a Th1 lymphocyte-mediated immune response. Thus, imiquimod's effect on the cell-mediated immune response is mediated by an indirect stimulation of the production of the Th1 cytokine IFN- .

An additional effect of imiquimod is its activation of Langerhans cells: it promotes their migration to the regional lymph node, thus potentially enhancing antigen presentation to $\mathrm{T}$ cells.

Imiquimod 5\% cream was initially approved in the US in 1997 as a topical treatment for external anogenital warts; after further clinical studies, it was approved in 2004 as a possible treatment for superficial basal cell carcinoma and actinic keratoses. ${ }^{8}$ Imiquimod's antitumorigenic effects are mediated by the up-regulation of local IFN- levels, suggesting that increased natural killer cell activity may be an important factor for both spontaneous and induced regression of basal cell carcinomas. ${ }^{2}$ Many studies have tested and discussed the use of imiquimod on several types of cutaneous tumors. Based on the results of previous studies, we reviewed all patients who had used imiquimod in our service.

\section{MATERIALS AND METHODS}

A retrospective study was designed to analyze the use of imiquimod on cutaneous tumors at the Dermatology Department of Hospital das Clinicas. A total of 574 patients were identified by the primary screening criterion: use of imiquimod in our hospital between January 2003 and October 2008 for HPV, pre-tumor, and tumor treatment. After review of the biopsy results for cutaneous tumors, 138 patients were retained for our analysis. Forty-two patients were excluded because of incomplete data, and seven were excluded because they were treated for lesions that were not tumors, such as actinic or seborrheic keratoses and warts. The records of 89 patients, corresponding to 123 lesions, were analyzed. Records of demographic data, biopsy results, treatment regimens, tumor outcomes, and adverse events were obtained. The data were entered into a spreadsheet (Excel) in order to tabulate descriptive statistics. The duration of treatment was calculated from the time a patient was initially prescribed topical $5 \%$ imiquimod cream until the time that the tumor was determined to be clinically cured or the treatment was stopped due to failure (minimum treatment threshold of six weeks). The administration frequency varied from five to seven times per week. The follow-up period was calculated from the date that the tumor was judged as cured or not (after treatment) to the last observation available in the clinical records. Response rates were delineated by clinical examination or by histological evaluation of punch biopsies obtained from affected tumor sites.

\section{RESULTS}

Of the 89 patients, $56.2 \%(n=50)$ were women, and $43.8 \%(n=39)$ were men. The ratio of women to men was 1.28:1. The majority of patients were white $(n=60)$. The mean age was 63.1 years (11-92 years). The 123 individual lesions studied were classified based on the tumor type and the associated comorbidity; the treatment duration in weeks, response to imiquimod, months of follow-up, and recurrence of lesions were analyzed. Patients were divided in two groups based on whether or not they had comorbidities.

A total of 54 tumors $(44.2 \%)$ were located on the face, $18(15 \%)$ on the back, $18(15 \%)$ on the trunk, $13(11 \%)$ on the legs, $10(8.4 \%)$ on the arms, six $(5 \%)$ on the neck, two $(1.5 \%)$ on the penis, one on the lower lip, and one ion the axillae $(0.08 \%$ each)

There were 29 lesions from 19 patients with comorbidities; these comorbidities included epidermodysplasia verruciformis $(n=7)$, albinism $(n=3)$, xeroderma pigmentosum $(n=1)$, Brooke-Spiegler syndrome $(n=2)$, basal cell nevus syndrome $(n=10)$, HIV $(n=1)$, chronic lymphocytic leukemia $(n=1), B-c e l l$ lymphoma $(n=2)$, and kidney transplantation $(n=2)$. The lesions found in patients with comorbidities included Bowen's disease $(n=6)$, superficial BCC $(n=6)$, superficial and nodular BCC $(n=6)$, aggressive (sclerodermiform or micronodular) basal cell carcinoma $(n=7)$, nodular BCC $(n=1)$ trichoepithelioma $(n=2)$, and erythroplasia of Queyrat $(n=1)$. Forty-five percent of these lesions $(n=13)$ showed no response to imiquimod, $17.5 \%(n=5)$ showed a partial response, and $38 \%$ $(n=11)$ showed a complete response. In terms of clinical or histologic cure, the cure rates were $50 \%(n=6)$ for Bowen's disease and $83.5 \%(n=6)$ for superficial BCC. Superficial and nodular BCC $(n=6)$ demonstrated no response and a partial response in $83.5 \%$ and $16.5 \%$ of patients with comorbidities, respectively. Aggressive BCC displayed no response to imiquimod in $71.5 \%$ of these patients and a complete response in $28.5 \%$. Erythroplasia of Queyrat presented a complete response to treatment, and nodular 
BCC and trichoepitheliomas demonstrated a partial response. No recurrence was detected, and patients who showed no response or only a partial response to treatment were treated with another therapeutic method soon after the use of imiquimod was terminated (Table 1).

There were 94 lesions from 70 patients without comorbidities; these lesions included superficial BCC $(n=51)$, superficial and nodular BCC $(n=21)$, nodular BCC $(n=6)$, aggressive (sclerodermiform or micronodular) BCC $(n=6)$, Bowen's disease $(n=7), \operatorname{SCC}(n=1)$, erythroplasia of Queyrat $(n=1)$, and Paget's disease $(n=1)$. Seventy-three percent of the patients without comorbidities demonstrated a complete response to treatment, and $27 \%$ showed no response.

The cure rates were $85.7 \%$ and $78.4 \%$ for nodular/ superficial basal cell carcinoma and superficial BCC, respectively. Among the cured patients, inclusion of the five patients with superficial BCC who were treated twice with imiquimod (12 weeks of use) raised the cure rate to $88 \%$. Paget's disease and erythroplasia of Queyrat presented a partial response to treatment. The cure rate was $57 \%$ for Bowen's disease and 50\% for nodular BCC. Surprisingly, mixed BCC with an aggressive sclerodermiform or micronodular component displayed a cure rate of $50 \%$, and one SCC lesion showed a complete response. One important finding was that, of the tumors considered clinically cured, none recurred during the extended follow-up period.

Patients who showed no response or only a partial response to treatment were treated via another therapeutic option. Thirty-seven lesions did not respond to imiquimod; they were subsequently treated with surgery $(n=15)$, photodynamic therapy $(n=10)$, imiquimod $(n=7)$, and cryotherapy $(n=1)$. Of these 37 lesions, 23 were from patients without comorbidities, and 14 were from patients with comorbidities; this corresponds to nearly half $(48.3 \%)$ of all tumors found on patients with comorbidities. Fourteen lesions $(37.8 \%)$ were located on the face, seven (19\%) on the back, five $(13.5 \%)$ on the neck, four $(10.8 \%)$ on the trunk, four $(10.8 \%)$ on the legs, two $(0.05 \%)$ on the arms, and one $(0.025 \%)$ on the lower lip. Among all the lesions located on the back, $38.8 \%$ recurred, compared to recurrence rates of $30.8 \%$ on the legs, $26 \%$ on the face, $22.2 \%$ on the trunk, and $20 \%$ on the arms. Tumors located on the penis and in theaxillae presented no recurrence, and the only lesion found on the lower lip recurred. Neck lesions exhibited the highest levels of recurrence: $83.3 \%$ for a total of six lesions. Eleven lesions were superficial BCC, nine were superficial and nodular $\mathrm{BCC}$, eight were mixed $\mathrm{BCC}$ with an aggressive component, six were Bowen's disease, and three were nodular BCC. Of all the aggressive BCC lesions, $61.5 \%$ recurred, compared to $42.8 \%$ of nodular BCC lesions, $41.5 \%$

Table 1 - Usage of topical 5\% imiquimod cream in patients with comorbidities

\begin{tabular}{|c|c|c|c|c|}
\hline Comorbidities/ Number of patients & $\begin{array}{l}\text { Histology/Number } \\
\text { of tumors }\end{array}$ & $\begin{array}{l}\text { Treatment duration } \\
\text { (median) }\end{array}$ & $\begin{array}{l}\text { Response to } \\
\text { treatment }\end{array}$ & Follow-up (median) \\
\hline \multirow[t]{3}{*}{$\begin{array}{l}\text { Epidermodysplasia Verruciformis } \\
(05)\end{array}$} & Bowen's disease (05) & 5.6 weeks & $\begin{array}{l}02 \text { complete; } 01 \text { partial; } \\
02 \text { none }\end{array}$ & 20 months \\
\hline & $\begin{array}{l}\text { Superficial/nodular } \\
\text { BCC }(01)\end{array}$ & 06 weeks & None & 14 months \\
\hline & Superficial BCC (01) & 04 weeks & Complete & No \\
\hline Albinism (02) & Superficial BCC (03) & 08 weeks & 02 complete; 01 none & 24.6 months \\
\hline Xeroderma Pigmentosum (01) & $\begin{array}{l}\text { Sclerodermiform } \\
\text { BCC -positive margin }(01)\end{array}$ & 08 weeks & Complete & 26 months \\
\hline Brooke-Spiegler syndrome $(02)$ & Trichoepithelioma (02) & 32 weeks & Partial & 10 months \\
\hline \multirow[t]{3}{*}{ Basal cell nevus syndrome (05) } & Nodular/sclerodermiform BCC (05) & 114 weeks & 05 none & 31 months \\
\hline & $\begin{array}{l}\text { Superficial/nodular } \\
\text { BCC }(04)\end{array}$ & 86,5 weeks & 01 partial; 03 none & 26 months \\
\hline & Nodular BCC (01) & 116 weeks & Partial & 24 months \\
\hline HIV (01) & Erythroplasia of Queyrat (01) & 03 weeks & Complete & 03 months \\
\hline Chronic lymphocytic leukemia (01) & Superficial/nodular BCC (01) & 06 weeks & No & 10 months \\
\hline Kidney transplantation (01) & $\begin{array}{l}\text { Bowen's disease }(01) \\
\text { Superficial/nodular/micronodular } \\
\text { BCC }(01)\end{array}$ & $\begin{array}{l}\text { Not available } \\
\text { Not available }\end{array}$ & $\begin{array}{l}\text { Complete } \\
\text { Complete }\end{array}$ & $\begin{array}{l}5 \text { months } \\
5 \text { months }\end{array}$ \\
\hline B-cell Lymphoma (01) & Superficial BCC (02) & 06 weeks & 02 complete & 12 months \\
\hline
\end{tabular}

BCC: basal cell carcinoma 
Table 2 - Usage of topical 5\% imiquimod cream in patients without comorbidities

\begin{tabular}{lcccc}
\hline Cutaneous disease/Number of patients & $\begin{array}{c}\text { Number of } \\
\text { tumors }\end{array}$ & $\begin{array}{c}\text { Treatment duration } \\
\text { (median) }\end{array}$ & $\begin{array}{c}\text { Response to } \\
\text { treatment }\end{array}$ & $\begin{array}{c}\text { Follow-up } \\
\text { (median) }\end{array}$ \\
\hline Paget's disease (01) & 01 & 24 weeks & Partial & 29 months \\
Erythroplasia of Queyrat (01) & 01 & 06 weeks & Partial & 26 months \\
Level 1 SCC (01) & 01 & 06 weeks & Complete & 01 month \\
Bowen's disease (07) & 07 & 6.1 weeks & 04 complete; 03 none & 15.4 months \\
Nodular BCC (05) & 06 & 8.2 weeks & 03 complete; 03 none & 10.3 months \\
Mixed BCC with an aggressive component of & 06 & 13.5 weeks & 03 complete, 03 none & 13.6 months \\
sclerodermiform or micronodular (06) & & & & 18 complete; 03 none \\
Superf/nodular BCC (18) & 21 & 7.3 weeks & 06.7 months \\
Superficial BCC (39) & 51 & 6.9 weeks & 39 complete; 12 none & 15.2 months \\
\hline BCC: ba
\end{tabular}

BCC: basal cell carcinoma; SCC: squamous cell carcinoma

of Bowen's disease lesions, 33.3\% of superficial and nodular lesions, and $19.3 \%$ of superficial BCC lesions (Table 2).

Of the seven patients who were treated with imiquimod twice, five presented a complete response after the second cycle of use, which brought the total duration of treatment to twelve weeks; the other two patients did not show any response. We were unable to determine the method used to treat three lesions that showed no response, and one patient refused to try another treatment after imiquimod failed. Patients with Paget's disease and erythroplasia of Queyrat showed a partial response and demonstrated good results after treatment with PDT.

Eleven patients did not have any local reactions, such as erythema, vesiculation, erosions, scaling, crusting, burning, and itching. These patients had a total of 15 tumors, eight of which $(53.3 \%)$ presented no response to imiquimod and had to be treated via other therapeutic methods. Two of these eight tumors were treated with another cycle of imiquimod, demonstrating a complete response after the second cycle of treatment.

The majority of patients presented mild (itching, erythema, and scaling) to moderate (intense erythema, crusting, vesicles, erosion) local skin reactions that were completely tolerable and healed spontaneously posttreatment. Only three patients experienced systemic reactions, such as nausea, headache, and flu-like symptoms; these side effects did not interfere with the treatment.

Two patients were subjected to curettage: one was affected by basal cell nevus syndrome and underwent curettage before the application of imiquimod, and the other underwent curettage after imiquimod treatment, as the tumor was $15 \mathrm{~cm}^{2}$ in size and presented crusting post-treatment. The first patient had two tumors (superficial and nodular $\mathrm{BCC}$ ) that demonstrated a partial response to imiquimod, and the second had one superficial BCC lesion that showed a complete response.
The potential use of imiquimod as an adjuvant treatment to surgery may warrant consideration. We described one case of sclerodermiform BCC in a patient affected by xeroderma pigmentosum with positive histologic lateral margins; the lesion demonstrated a complete response to imiquimod treatment.

Another goal of our study was to learn about the healing patterns after treatment with 5\% imiquimod cream. In almost all patients, imiquimod led to good cosmetic outcomes; the skin on the cured area usually displayed a normal appearance, without scarring or with only mild erythema, atrophy, or depigmentation. Some small persistent scars were likely due to the initial biopsy; they were almost imperceptible.

\section{DISCUSSION}

Imiquimod has been recognized as a topical treatment for anogenital warts since 1997 and for superficial BCC and actinic keratoses since 2004.

In previous studies of superficial BCC in which the tumor site was excised after treatment to confirm tumor status, histologic clearance rates ranged from $79 \%$ to $82 \%$ with imiquimod application five or seven times per week for six weeks. ${ }^{4}$

Another paper reported a cure rate of $90 \%$ for superficial BCC. ${ }^{12}$ Our experience revealed initial clinical or histologic clearance rates for superficial BCC of $78.4 \%$. Eleven patients did not respond to imiquimod, five of whom were treated again with another course of six weeks and presented a complete response to imiquimod during the second course. Classifying these five lesions as cured raised the cure rate of superficial BCC to $88 \%$. Three superficial BCC lesions were treated for twelve or fourteen weeks; one was treated for six weeks, and two were treated irregularly.

A $71 \%$ histologic clearance rate was reported in a study 
of nodular BCC in which imiquimod was applied five times per week. ${ }^{5}$ Our initial clinical tumor cure rates were $85.7 \%$ for superficial and nodular BCC and 50\% for nodular BCC, similar to previously reported results; together, these results support the use of imiquimod to treat these type of tumors.

The overall initial tumor clinical cure rate reported in another paper was $93.4 \%$, in a study including 69 nodular BCC, 36 SCC, six basosquamous, and 11 morpheaform tumors. ${ }^{8}$ The overall cure rate for our patients without comorbidities was 73\%; this included 51 superficial BCC, six nodular BCC, 21 superficial and nodular BCC, six mixed BCC with an aggressive component, one SCC, and seven Bowen's disease lesions. Somewhat surprisingly, one study revealed a $100 \%$ clinical tumor cure rate for SCC and sclerodermiform BCC. ${ }^{8}$ The clinical cure rates for our patients without comorbidities, even for high-risk tumors such as mixed aggressive BCC (cure rate of 50\%) and SCC (complete response in one tumor), were considerable; these results support the usage of topical imiquimod to treat these types of lesions in special situations.

Yet, in contrast to the majority of the studies already published in the literature, which predominantly limited imiquimod treatment to superficial BCC and low-risk tumors, we intended to include the whole spectrum of cutaneous tumors and comorbidities. By doing so, we observed clear differences in response rates and were able to describe the use of imiquimod in trichoepitheliomas, erythroplasia of Queyrat, and Paget's disease, all with a complete or partial response. There are three cases of Paget's disease treated with imiquimod have been reported in the literature (two women and one man), with all patients showing complete response.

One patient whose trichoepitheliomas were treated with imiquimod and retinoic acid for three years presented $80 \%$ clearance. There are five papers describing the use of imiquimod on erythroplasia of Queyrat. Three patients, including one with HIV, showed complete response. These data, including the experiences that we describe at our dermatology service, support the use of imiquimod in the treatment of these lesions.

Patients with epidermodysplasia verruciformis, basal cell nevus syndrome, xeroderma pigmentosum, and albinism were described in our study. We observed that these particular types of patients presented a worse response to imiquimod for the treatment of aggressive BCC, nodular BCC, and Bowen's disease. Three studies, comprising 49, 15 , and five patients with Bowen's disease, reported an $86 \%$, $73 \%$, and $80 \%$ complete response, respectively; these results differ from those of our study, in which the cure rate with imiquimod was $57 \%$.

Lesions located on the neck, back, or legs and those that did not present a local inflammatory reaction also presented lower cure rates. Perhaps a deficiency or a decreased number of toll-like receptors at these sites explain this lower response; this may occur, for example, due to alterations in inflammatory cascades. More studies are needed to understand the real cause of this difference in response.

The cure rate for lesions on patients with comorbidities was $38 \%$, compared to $73 \%$ for patients without comorbidities. However, even for patients with comorbidities, low-risk tumors such as superficial BCC and Bowen's disease presented excellent cure rates of $86.5 \%$ and $50 \%$, respectively; these results seem to favor histology as an important prognostic parameter. Superficial and nodular, nodular, mixed and sclerodermiform BCC showed low cure rates, especially in patients with basal cell nevus syndrome. None of these patients demonstrated a complete response to imiquimod, in contrast to the data found in the literature showing cure with imiquimod in patients with basal cell nevus syndrome. However, we demonstrated cure of some lesions in patients with epidermodysplasia verruciformis, which has not been reported in the previous literature, and in patients with xeroderma pigmentosum (already described).

There are no reports of imiquimod treatment in patients with albinism. We also observed that patients with cutaneous comorbidities (basal cell nevus syndrome, epidermodysplasia verruciformis, albinism, xeroderma pigmentosum, and Brooke-Spiegler syndrome) showed a diminished response to treatment compared to patients with other types of comorbidities, such as hematologic neoplasia, immunosuppression for kidney transplantation, and HIV. Therefore, the use of imiquimod in patients with cutaneous comorbidities needs to be reviewed based on the specific circumstances of each patient.

Worse outcomes were also observed for tumors that did not show local reactions: more than half of them displayed no response to imiquimod. However, even lesions without a local reaction can be repeatedly treated with imiquimod and show a complete response during the second course of treatment. Seven lesions, including two that did not present a local reaction, were treated with imiquimod for a second cycle of six weeks, and $71.5 \%$ presented a complete response during this second course. Therefore, imiquimod can be used repeatedly if there is no response in the first cycle of treatment; this is especially true for low-risk tumors.

The main advantage of topical treatment of cutaneous tumors with $5 \%$ imiquimod cream is that it provides an option for patients with contraindications to surgery or patients with multiple lesions. Furthermore, imiquimod is a relatively low-cost treatment with tolerable side effects and a high cure rate, especially for patients without comorbidities and with low-risk tumors; these patients generally have a 
better prognosis. However, in selected cases of patients with cutaneous comorbidities or high-risk tumors, imiquimod is also considered as a supportive therapeutic method.

\section{CONCLUSIONS}

With the increasing incidence of cutaneous tumors, especially BCC, imiquimod may offer an alternative treatment option that avoids surgical or ablative therapy, particularly in patients who present a challenge to surgery (the elderly, patients on anticoagulation medication, multimorbid patients, patients with multiple lesions, and patients with recurrent or incompletely excised tumors).
Imiquimod presents an alternative for diseases traditionally treated by surgery, and it may be an important option in the management of increasingly common cutaneous malignancies. For patients without comorbidities, our study revealed good cure rates for superficial BCC and superficial/nodular BCC (88\% and $85 \%$, respectively). Nodular and aggressive $\mathrm{BCC}$ and Bowen's disease exhibited lower cure rates $(50 \%, 50 \%$, and $57 \%$, respectively). Imiquimod is also considered as a good alternative for Paget's disease, erythroplasia of Queyrat, and trichoepitheliomas. For patients with cutaneous comorbidities, imiquimod is a good option only for low-risk tumors.

\section{REFERENCES}

1. Gaspari AA, Sauder DN. Immunotherapy of basal cell carcinoma: evolving approaches. Dermatol Surg. 2003;29:1027-34.

2. Sullivan TP, DeAraujo T, Vincek V, Berman B. Evaluation of superficial basal cell carcinomas after treatment with imiquimod 5\% cream or vehicle for apoptosis and lymphocyte phenotyping. Dermatol Surg. 2003;29:1181-6.

3. Sterry S, Ruzicka T, Herrera E, Takwale A, Bichel J, Andres K, et al. Imiquimod 5\% cream for the treatment of superficial and nodular basal cell carcinoma: randomized studies comparing low frequency dosing with and without occlusion. Br J Dermatol. 2002;147:1227-36.

4. Geisse J, Caro I, Lindholm J, Golitz L, Stampone P, Owens M. Imiquimod 5\% cream for the treatment of superficial basal cell carcinomas: results from two phase III, randomized, vehicle-controlled studies. J Am Acad Dematol. 2004;50: 722-33.

5. Schulze HJ, Cribier B, Requena L, Reifenberger J, Ferrándiz C, Garcia Diez A, et al. Imiquimod 5\% cream for the treatment os superficial basal cell carcinoma: results from a randomized vehicle-controlled phase III study in Europe. Br J Dermatol. 2005;152:939-47.

6. Shumack S, Robinson J, Kossard S, Golitz L, Greenway H, Schroeter A, et al. Efficacy of topical 5\% imiquimod for the tyreatment of nodular basal cell carcinoma: comparison of dosing regimes. Arch Dermatol. 2002;138:1165-71.

7. Schiessl C, Wolber C, Tauber M, Offner F, Strohal R. Treatment of all basal cell carcinoma variants including large and high-risk lesions with 5\% imiquimod cream: histological and clinical changes, outcome, and follow-up. J Drugs Dermatol 2007;6:507-13.

8. Warshauer E, Warshauer BL. Clearance of basal cell and superficial squamous cell carcinomas after imiquimod therapy. J Drugs Dermatol 2008; 7: 447-51.
9. Cowen E, Mercurio MG, Gaspari AA. An open case series of patients with basal cell carcinoma treated with topical 5\% imiquimod cream. J Am Acad Dermatol. 2002;47:240-8.

10. Drehs MM, Cook-Bolden F, Tanzi EL, Weinberg JM. Successful treatment of multiple superficial basal cell carcinomas with topical imiquimod: case report and review of the literature. Dermatol Surg. 2002;28:427-9.

11. Micali G, De Pasquale R, Caltabiano R, Impallomeni R, Lacarrubba F. Topical imiquimod treatment of superficial and nodular basal cell carcinomas in patients affected by basal cell nevus syndrome: a preliminary report. J Dermatol Treatment. 2002;13:123-7.

12. Gollnick H, Barona CG, Frank RG, Ruzicka T, Megahed M, Tebbs $\mathrm{V}$, et al. Recurrence rate of superficial basal cell carcinoma following successful treatment with imiquimod 5\% cream: interim 2-year results from an ongoing 5-year follow-up study in Europe. Eur J Dermatol. 2005; 15:374-81.

13. Festa Neto C. Tratamento tópico do carcinoma basocelular superficial e nodular pelo imiquimode creme a 5\%: observação de 10 casos. An Bras Dermatol. 2002;77:693-8.

14. Beutner KR, Geisse JK, Helman D, Fox TL, Ginkel A, Owens ML. Therapeutic response of basal cell carcinoma to the immune response modifier imiquimod 5\% cream. J Am Acad Dermatol. 1999;1:1002-7.

15. Marks R, Gebauer K, Shumack S, Amies M, Bryden J, Fox TL, et al. Imiquimod 5\% cream in the treatment of superficial basal cell carcinoma: results of a multicenter 6-week dose multiple response trial.J Am Acad Dermatol. 2001; 44:807-13.

16. Geisse J, Caro I, Lindholm J, Golitz L, Stampone P, Owens M. Imiquimod 5\% cream for the treatment of superficial basal cell carcinoma: Results from two phase, randomized, vehicle-controlled studies. J Am Acad Dermatol. 2004;50: 722-33. 